\title{
COMMENTARY
}

\section{Macrolides and community-acquired pneumonia: is quorum sensing the key?}

\author{
Matt P Wise ${ }^{1 *}$, David W Williams², Michael AO Lewis² and Paul J Frost ${ }^{1}$
}

\begin{abstract}
Combination therapy with two antimicrobial agents is superior to monotherapy in severe communityacquired pneumonia, and recent data suggest that addition of a macrolide as the second antibiotic might be superior to other combinations. This observation requires confirmation in a randomised control trial, but this group of antibiotics have pleiotropic effects that extend beyond bacterial killing. Macrolides inhibit bacterial cell-to-cell communication or quorum sensing, which not only might be an important mechanism of action for these drugs in severe infections but may also provide a novel target for the development of new anti-infective drugs.
\end{abstract}

Outcome in community-acquired pneumonia (CAP) is adversely affected by increasing severity of illness, comorbidity and age. Organisational factors such as timely administration of appropriate antibiotics, prompt admission to critical care and adherence to antibiotic policies, however, are also important in influencing outcome [1-3]. Combination therapy with two antimicrobial agents seems superior to monotherapy in severe CAP, and this approach is recommended by a number of organisations [4,5]. The Infectious Diseases Society of America/American Thoracic Society guidelines suggest therapy with a $\beta$-lactam antibiotic, with the addition of either a macrolide or fluoroquinolone antibiotic [4], whilst the British Thoracic Society recommends initiating a $\beta$-lactam/macrolide antibiotic combination [5].

Martin-Loeches and colleagues recently conducted a prospective, observational cohort, multicentre study involving 218 mechanically ventilated CAP patients to see what effect different antibiotic combinations had on mortality [6]. These investigators reported that the

*Correspondence: mattwise@doctors.org.uk

'Adult Critical Care, University Hospital of Wales, Cardiff CF14 4XW, UK

Full list of author information is available at the end of the article addition of a macrolide, but not a fluoroquinolone, to standard antibiotic therapy was associated with reduced mortality in patients admitted to critical care with CAP. Death in critical care occurred in $26.1 \%$ of individuals receiving combination therapy with a macrolide, compared with $46.3 \%$ in those receiving fluoroquinolones [6]. These results support data from other observational studies that suggest $\beta$-lactam/macrolide combinations offer a survival advantage in severe CAP. This body of data is not scientifically robust enough, however, to adequately answer the question of whether adding a macrolide to a $\beta$-lactam confers a survival advantage this will only be satisfactorily addressed by a large prospective randomised control trial.

In addition to activity against atypical bacteria, macrolides have ubiquitous immunomodulatory effects. Speculating how this group of drugs might offer a survival advantage when added to a $\beta$-lactam is therefore of interest, and several plausible mechanisms exist. Treatment of undiagnosed atypical pneumonia could occur since $53 \%$ of patients in the reported study had no microbiological diagnosis [6]; however, this seems unlikely as one might expect fluoroquinolones to be equally effective [7]. Moreover, studies limited to pneumococcal disease demonstrate that addition of a macrolide improves survival [8]. It also seems improbable that synergistic killing is responsible, as equivalency with fluoroquinolones would be expected.

Many researchers have focused on the pleiotropic immunomodulatory effects [9] observed with macrolides as the reason why these agents may be beneficial in CAP. Macrolides, at doses lower than those required for antibacterial activity, alter the production of cytokines and chemokines, and reduce cellular infiltrates and mucous production [9]. The immunomodulatory effects of macrolides are illustrated by diffuse panbronchiolitis. A chronic progressive lung disease found largely in Japan, diffuse panbronchiolitis is characterised by mixed restrictive and obstructive pulmonary function, interstitial infiltrates and Pseudomonas aeruginosa infection. Long-term, low-dose macrolide treatment improves lung function and increases 10-year survival rates from around 15 to $90 \%$ [9]. 
Macrolides are now being explored in new therapeutic strategies for a wide range of pulmonary and extrapulmonary conditions, including asthma, cystic fibrosis, rhinosinusitis, inflammatory bowel disease, psoriasis and rosacea [9]. Clearly immunomodulatory effects could be important in altering mortality in CAP, but these drugs also have direct effects on bacteria through inhibiting quorum sensing.

Quorum sensing describes bacterial cell-to-cell communication that occurs as a function of changing cell density. These communication pathways are important in the pathogenesis of bacterial species causing human disease, including Staphylococcus aureus, Streptococcus pneumoniae, Escherichia coli and P. aeruginosa [10,11]. Quorum-sensing bacteria produce and release signal molecules or autoinducers, which regulate gene expression within the bacterial population and are closely linked to both biofilm formation and expression of virulence factors. Biofilms are structured populations of bacteria within a polysaccharide matrix, and these growth forms are more resistant to antibiotics. The discovery of biofilms as an entity did not occur until the late 1970 s, and they are often still only considered in the context of chronic or device-associated infections; however, pneumonia caused by $S$. pneumoniae exists as a biofilm in lung tissue [11]. Acute bacterial infections associated with biofilm formation might also be relatively common. One of the diagnostic criteria for biofilm infection is a culture-negative result despite a clinically documented infection [12], a situation encountered in 30 to $50 \%$ of severe sepsis and septic shock [6].

Macrolides at subminimum inhibitory concentrations have been demonstrated to antagonise quorum sensing in $P$. aeruginosa, resulting in diminished virulence, biofilm formation and oxidative stress response [13]. Significantly, inhibition of quorum sensing reduces pathogenicity of bacteria and impedes formation of antibiotic-resistant biofilms, and therefore offers an attractive mechanism whereby the addition of a macrolide could reduce mortality in CAP [6]. If macrolides do confer additional efficacy because of immunomodulatory effects or inhibition of quorum sensing, or both, one might expect them to be an effective therapeutic strategy applicable to many other infections encountered in critically ill patients. Indeed, the addition of clarithromycin to patients with ventilator-associated pneumonia accelerated resolution of pneumonia and weaning from mechanical ventilation [14].

It may be possible to approach the question of whether immunomodulation or inhibition of quorum sensing is more important in reducing mortality experimentally. Lesprit and colleagues described the important role of $P$. aeruginosa quorum sensing in rat pulmonary infection using the virulent wild-type strain $P$. aeruginosa PAO1 and the less virulent mutant strain $P$. aeruginosa PAOR with a deficient quorum-sensing pathway [15]. Using this model system it would be beneficial to examine whether macrolides act predominantly through disrupting quorum sensing, as one would then expect to see little reduction in mortality caused by a large inoculum of the mutant PAOR but a significant effect on pneumonia caused by a smaller dose of the wild-type PAO1.

At a time when few new antimicrobial agents are being commercially developed for clinical use and the burden of infection caused by multiresistant bacteria is increasing, the need for novel approaches to the management of infection is essential. Quorum sensing determines both bacterial virulence and biofilm formation; it is a common pathway for pathogens and represents an attractive new target for the development of drugs in the fight against infection [10].

\section{Abbreviations}

CAP, community-acquired pneumonia.

\section{Competing interests}

The authors declare that they have no competing interests.

\section{Author details}

'Adult Critical Care, University Hospital of Wales, Cardiff CF14 4XW, UK. 2School of Dentistry, Cardiff University, Cardiff CF14 4XY, UK.

Published: 20 July 2010

\section{References}

1. Lipman J, Boots R: A new paradigm for treating infections 'go hard and go home'. Crit Care Resusc 2009, 11:276-281.

2. Restrepo MI, Mortensen EM, Rello J, Brody J, Anzueto A: Late admission to the ICU in patients with community-acquired pneumonia is associated with higher mortality. Chest 2010, 137:552-557.

3. McCabe C, Kirchner C, Zhang H, Daley J, Fisman DN: Guideline-concordant therapy and reduced mortality and length of stay in adults with community-acquired pneumonia: playing by the rules. Arch Intern Med 2009, 169:1525-1531.

4. Mandell LA, Wunderink RG, Anzueto A, Bartlett JG, Campbell GD, Dean NC, Dowell SF, File TM, Musher DM, Niederman MS, Torres A, Whitney CG; Infectious Diseases Society of America; American Thoracic Society: Infectious Diseases Society of America/American Thoracic Society consensus guidelines on the management of community acquired pneumonia in adults. Clin Infect Dis 2007, 44(Suppl 2):S27-S72.

5. Lim WS, Baudouin SV, George RC, Hill AT, Jamieson C, Le Jeune I, Macfarlane JT, Read RC, Roberts HJ, Levy ML, Wani M, Woodhead MA; Pneumonia Guidelines Committee of the BTS Standards of Care Committee: BTS guidelines for the management of community acquired pneumonia in adults: update 2009. Thorax 2009, 64(Suppl 3):iii1-iii55.

6. Martin-Loeches I, Lisboa T, Rodriguez A, Putensen C, Annane D, GarnachoMontero J, Restrepo Ml, Rello J: Combination antibiotic therapy with macrolides improves survival in intubated patients with communityacquired pneumonia. Intensive Care Med 2010, 36:612-620.

7. Roig J, Casal J, Gispert P, Gea E: Antibiotic therapy of community-acquired pneumonia (CAP) caused by atypical agents. Med Mal Infect 2006, 36:680-689.

8. Baddour LM, Yu VL, Klugman KP, Feldman C, Ortqvist A, Rello J, Morris AJ, Luna CM, Snydman DR, Ko WC, Chedid MB, Hui DS, Andremont A, Chiou CC; International Pneumococcal Study Group: Combination antibiotic therapy lowers mortality among severely ill patients with pneumococcal bacteraemia. Am J Respir Crit Care Med 2004, 170:400-404.

9. Shinkai M, Henke MO, Rubin BK: Macrolide antibiotics as immunomodulatory medications: proposed mechanisms of action. Pharmacol Ther 2008, 117:393-405.

10. Njoroge J, Sperandio V: Jamming bacterial communication: new 
approaches for the treatment of infectious diseases. EMBO Mol Med 2009, 1:201-210

11. Oggioni MR, Trappetti C, Kadioglu A, Cassone M, lannelli F, Ricci S, Andrew PW, Pozzi G: Switch from planktonic to sessile life: a major event in pneumococcal pathogenesis. Mol Microbiol 2006, 61:1196-1210.

12. Hall-Stoodley L, Stoodley P: Evolving concepts in biofilm infections. Cell Microbiol 2009, 11:1034-1043.

13. Nalca Y, Jansch L, Bredenbruch F, Geffers R, Buer J, Haussler S: Quorumsensing antagonistic activities of azithromycin in Pseudomonas aeruginosa PAO1: a global approach. Antimicrob Agents Chemother 2006, 50:1680-1688.

14. Giamarellos-Bourboulis EJ, Pechere JC, Routsi C, Plachouras D, Kollias S, Raftogiannis M, Zervakis D, Baziaka F, Koronaios A, Antonopoulou A, Markaki V, Koutoukas P, Papadomichelakis E, Tsanganos T, Armaganidis A, Koussoulas
V, Kotanidou A, Roussos C, Giamarellou H: Effect of clarithromycin in patients with sepsis and ventilator-associated pneumonia. Clin Infect Dis 2008, 46:1157-1164.

15. Lesprit P, Faurisson F, Join-Lambert O, Roudot-Thoraval F, Foglino M, Vissuzaine C, Carbon C: Role of the quorum-sensing system in experimental pneumonia due to Pseudomonas aeruginosa in rats. Am J Respir Crit Care Med 2003, 167:1478-1482.

\section{doi:10.1186/cc9084}

Cite this article as: Wise MP, et al:: Macrolides and community-acquired pneumonia: is quorum sensing the key? Critical Care 2010, 14:181. 